Volume 8, No.1.4, 2019

International Journal of Advanced Trends in Computer Science and Engineering

Available Online at http://www.warse.org/IJATCSE/static/pdf/file/ijatcse4381.42019.pdf

https://doi.org/10.30534/ijatcse/2019/4381.42019

\title{
Performance Evaluation of an Adaptive Forwarding Strategy in Named Data Networking
}

\author{
Raja Azlina Raja Mahmood ${ }^{1}$, Nor Faizah Jemangin ${ }^{2}$, Masnida Hussin ${ }^{3}$ \\ ${ }^{1,2,3}$ Faculty of Computer Science and Information Technology, Universiti Putra Malaysia, \\ ${ }^{1}$ raja_azlina@upm.edu.my \\ 2nfaizj@gmail.com \\ 33masnida@upm.edu.my
}

\begin{abstract}
Named Data Networking (NDN) is an envisioned Internet architecture that uses named data to locate the data of interest as opposed to the IP address of the stored data. The forwarding strategy is critical in this network to ensure the data is well received in a timely manner. Stochastic Adaptive Forwarding (SAF) is said to have an increased throughput and provide quick recovery as it efficiently chooses the potential forwarding link whenever there is a failure on the existing link. SAF is designed to consider both the context and content of the networks to optimize its forwarding behavior. This paper compares the performance of SAF and Best Route algorithms, in terms of Interest satisfaction ratio, cache hit ratio, delay, Interest retransmission rate and hop count value. The results show SAF outperforms Best Route except for the hop count value.
\end{abstract}

Key words : Best Route, forwarding strategy, Named Data Networking, Stochastic Adaptive Forwarding.

\section{INTRODUCTION}

Over the years, a lot of effort has been made to improve the existing IP network performance, including Content Delivery Network (CDN), load balancers and cloud services to optimize the content delivery. CDN for example, is an effective solution to date but it is not able to optimize the bandwidth usage in local area network (LAN) or in wide area network (WAN), as the data is located in one's region data center. Hence, it has the potential to increase the risk of bottleneck in the network gateways should all nodes need to go to the endpoint of the network if they want to access the same content. Of recent, Named Data Networking (NDN) has been introduced to efficiently handling the content delivery.

NDN is a proposed future Internet architecture that shifts the network communication model from host-centric to data-centric. That is, it changes the network service orientation from delivering the packet to a given destination address to fetching the data identified by a given name, also known as data name [1]. Forwarding strategy determines whether, when, and where to forward each of the requested packet or also known as Interest packet. An adaptive forwarding strategy learns current network status through its interfaces to improve future decisions while a static forwarding strategy only relies on the decisions made by the routing protocol and unable to adapt with network changes. Stochastic Adaptive Forwarding (SAF) [2], a probability-based forwarding strategy aims to maximize the Interest, hence the Data satisfaction ratio while taking the delay, hop-count and transmission cost into considerations. It employs intelligent multi-path transmission using redundant paths as well as exploits content-based information to further improve its forwarding decisions.

In this paper, we compare SAF with Best Route algorithm to evaluate the Interest satisfaction ratio, cache hit ratio as well as hop count performance, which are similar to the work of [2]. The contribution of this paper is that we have included other metrics as well, which are the delay and Interest retransmission values, to fully understand SAF's performance. We also study the impact of having fixed number of servers as well as having gradually increased number of servers in the networks.

The remainder of this paper is organized as follows. Section 2 discusses NDN followed by existing NDN forwarding implementation in Section 3. Section 4 describes SAF design. Section 5 presents some of adaptive forwarding strategies in NDN. Section 6 provides the simulation parameters and scenarios used in this work. Section 7 discusses the results and finally, Section 8 concludes our findings with some potential future work remark.

\section{NAMED DATA NETWORKING}

Communication in NDN is driven by the exchange of two types of packets, Interest and Data. A consumer puts the name of a desired piece of data into an Interest packet and sends it to the network. Routers forward the Interest towards the data producer(s). Node with the requested data will return 
a Data packet that contains both the name and content, together with a signature by the producer's key that binds the two. Figure 1 shows the basic flow of Interest and Data processing in NDN [3]. Each NDN router maintains a Pending Interest Table (PIT), a Forwarding Information Base $(F I B)$ and a Content Store $(C S)$ together with its preferred forwarding strategy module. The process flow of Data packets has always taken the reverse path of the Interest packets and is summarized as follows, with details in [1]:

a. When a router received an Interest, it checks if it has the request data in its CS. If yes, it returns the Data packet on the interface from which the Interest came. If no matching entry exists, the router forwards the Interest toward the data producer(s) based on the information in the FIB and its forwarding strategy.

b. When a Data packet arrives, the router checks the PIT match entry and forwards the data to all downstream interfaces stated in that PIT entry. That particular PIT entry is then removed, and the router caches the Data in the CS.
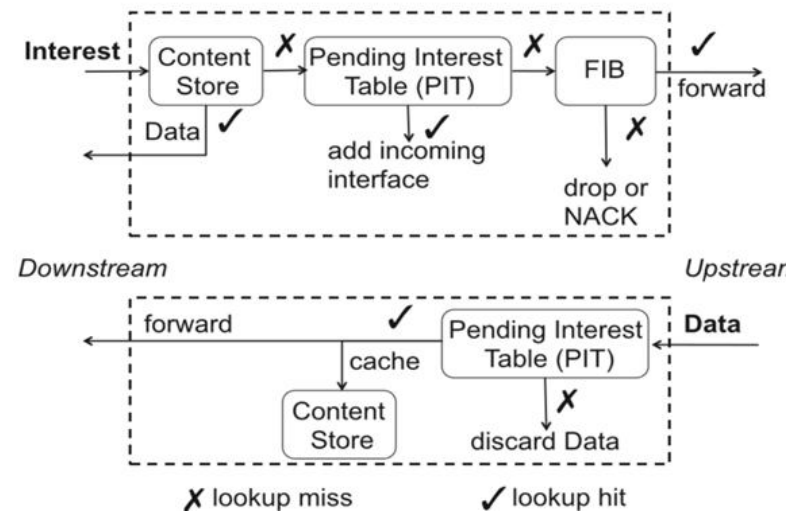

Figure 1: Interest and Data Processing in NDN [1]

\section{ROUTING AND FORWARDING}

NDN routes and forwards packets based on names and thus eliminate the existing IP address issues which include address space exhaustion, NAT traversal and address management [1]. NDN routers that employ conventional routing protocols such as link state and distance vector announce name prefixes that cover the data they are willing to serve, as opposed to the traditional IP prefixes. The routing protocol then propagates these announcements across the networks while constructing the FIBs. PIT records each pending Interest and the incoming interface(s), and removing the Interest after the matching Data is received or a timeout occurs. The employed adaptive forwarding strategy module then makes an informed decision to efficiently handling the Interests, including deciding which Interests to forward to which interfaces, prioritizing the different type of Interests and load-balancing the Interests among multiple interfaces [3], [4].

\section{STOCHASTIC ADAPATIVE FORWARDING}

Figure 2 shows an NDN node using SAF. Each node maintains a Forwarding Table (FWT), a two-dimensional matrix with rows that correspond to the set of faces and columns correspond to the different contents. The elements of the matrix are the confidence (probability) which a certain outgoing face or also known as path can provide data for a certain content prefix/type.

FWT performs adaptive forwarding through its Adaptation Engine. Modification of the probabilities within a single column, denoted by a red line, changes the forwarding probabilities for Interests asking for a specific content. These updates modify which faces/paths are preferred for forwarding Interests. Secondly, shifting forwarding probabilities among the columns of blue lines allows prioritization of specific content types/prefixes. For example, assume $c_{m}$ is deemed more important than $c_{k}$, thus the probability of dropping Interests for $c_{k}$ has increased. In summary, the forwarding strategy by SAF takes into account the context and content of the networks for optimal Interest/Data ratio performance. The detailed algorithm and discussion can be found in [2].

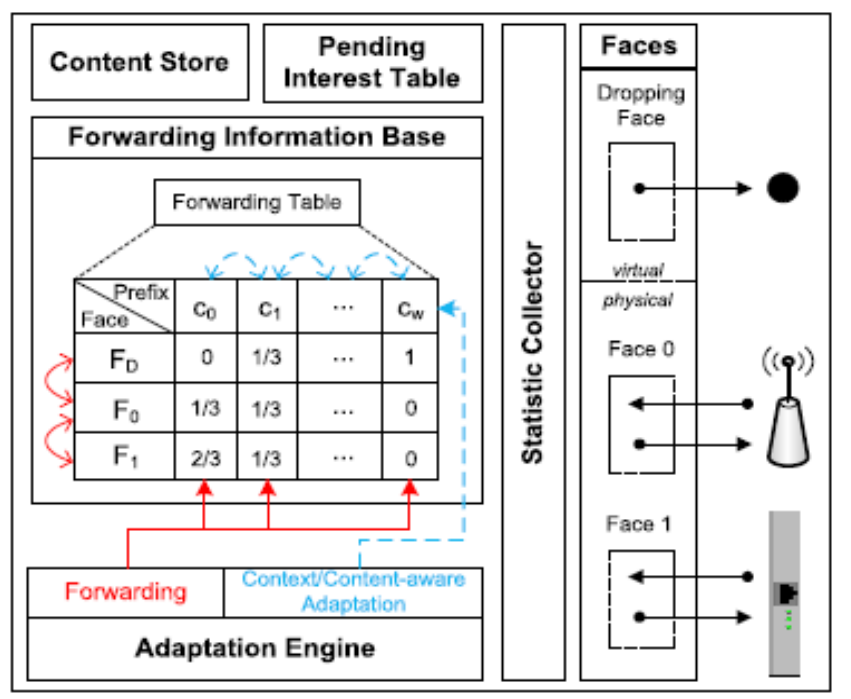

Figure 2: The model of an NDN node using SAF [2]

\section{RELATED WORKS}

The followings are some related works on adaptive forwarding strategy that aims to overcome congestion problems due to bottleneck or link failure. Moll, Posch and Hellwagner [5] introduced Persistent Interest (PI) that uses probing to identify alternative links in order to adapt with the changing conditions. However, it has high overhead in probing.

Few researchers use round trip time (RTT) as a basis indication to find alternative links. These include 
Raja Azlina Raja Mahmood et al., International Journal of Advanced Trends in Computer Science and Engineering, 8(1.4), 2019,281 - 287

Heterogeneous-Latency Adaptive Forwarding (HLAF) by [6], adaptive forwarding strategy with quality of service (QoS-FS) by [7], Adaptive SRTT Update (ASU) by [8], Probability-based Adaptive Forwarding (PAF) by [9] and On-demand Multi-Path Interest Forwarding (OMP-IF) by [10].

HLAF chooses the forwarding interfaces based on clear congestion signals such as Negative Acknowledgement (NACK) and RTT. Meanwhile QoS-FS uses exploration in FIB and then exploitation phase based on the required QoS in the interface. However, it was compared to Best Route which is not suitable as it is not the same type of forwarding strategy which manage QoS. ASU [8] is the first paper illustrated the effect of outdated forwarding states in the current scheme in interface ranking: probing oscillation and SRTT slow convergence. However, it only focused on interface ranking and does not impact the strategy of managing congestion and NACK. PAF uses Ant Colony Optimization (ACO) and calculate pheromone table via the RTT metric and selects the interfaces based on an RTT distribution. It utilizes the link that has a lower RTT. However, RTT is not a perfect metric to assess the performance of a link [11]. Consider a situation in which the nearby peer may be having less uploading capability than other peers and servers. And hence, merely using RTT values is not accurate [6]. OMP-IF uses multiple node disjoint paths for Interest forwarding simultaneously. Each router uses a single face for forwarding per content-prefix to ensure node disjointness. The multipath transmission is then trigged based on the path delays values using a weighted round-robin mechanism. However, some of the network resources would be left unused in this node disjoint path's approach [10].

The Partially observable Markov decision process (POMDP) has been designed to get the minimal cost for content delivery [12]. Potential decisions can be done without having complete information of the network state. However, high complexity in computational of the proposed POMDP solution makes it difficult to be implemented in real world.

Lei, Yuan and Wang [13] use Entropy-Based Probabilistic Forwarding (EPF) to calculate interface forwarding potential and availability in order to get the probability values of all interfaces. An interface with higher availability means more possibility it used to forward the Interests. This strategy has better load balance and throughput as compared to strategies which used interface selection by deterministic. However, EPF is computationally expensive to be implemented.

Best Route algorithm is a forwarding strategy that has been implemented in ndnSIM since version 1.0 and is still supported in latest version. Interests are forwarded to available faces, in ranks. The Interest will be sent to the highest-ranked Green face if it is available. If not, it will be forwarded to the highest-ranked Yellow face. If there are no available Green or Yellow faces, Interest is dropped [4]. It uses lowest-cost, mainly hop count preference [14]. In this preliminary study, we only compare these two forwarding strategies.

\section{SIMULATION PARAMETERS AND SCENARIOS}

The simulation has been implemented on Ubuntu 16.04 using ndnSIM 2.5 simulator [15] which based on NS-3 simulator platform. Table 1 shows the simulation parameters used in this work.

Table 1: Simulation Parameters

\begin{tabular}{|c|c|c|c|}
\hline $\begin{array}{l}\text { Infrastructure } \\
\text { Model }\end{array}$ & \multicolumn{3}{|c|}{$\begin{array}{l}\text { Large ISP network, interconnecting several } \\
\text { autonomous systems and access networks }\end{array}$} \\
\hline $\begin{array}{l}\text { Simulation } \\
\text { Tool }\end{array}$ & \multicolumn{3}{|l|}{ NS3/ndnSIM } \\
\hline $\begin{array}{l}\text { Network } \\
\text { Topology } \\
\text { Generator }\end{array}$ & \multicolumn{3}{|c|}{$\begin{array}{l}\text { BRITE - to build scale-free network in top } \\
\text { down- fashion }\end{array}$} \\
\hline Top level & \multicolumn{3}{|c|}{5 autonomous systems (AS) } \\
\hline $\begin{array}{l}\text { Each AS } \\
\text { maintains }\end{array}$ & \multicolumn{3}{|c|}{$\begin{array}{l}20 \text { nodes (bottom level) acting as ICN } \\
\text { routers } \\
\text { Hence, } 5 \text { × } 20=100 \text { ICN routers and } \\
\text { serving as access nodes for later on added } \\
\text { client and server nodes. }\end{array}$} \\
\hline $\begin{array}{l}\text { Client and } \\
\text { Servers }\end{array}$ & \multicolumn{3}{|c|}{$\begin{array}{l}\text { Randomly placed: } \\
\mathrm{a}=100 \text { clients } \\
\mathrm{b}=10 \text { servers or } \mathrm{c}=2,4,6,8 \text { and } 10 \text { servers }\end{array}$} \\
\hline $\begin{array}{l}\text { Topology } \\
\text { Model }\end{array}$ & \multicolumn{3}{|c|}{$\begin{array}{l}\text { Barabási-Albert model to generate } \\
\text { scale-free network }\end{array}$} \\
\hline \multirow[t]{2}{*}{$\begin{array}{l}\text { Link } \\
\text { bandwidth }\end{array}$} & Identifier & $\begin{array}{l}\text { Top level } \\
\text { (AS) }\end{array}$ & $\begin{array}{c}\text { Bottom } \\
\text { level } \\
\text { (Nodes) }\end{array}$ \\
\hline & MediumBw & $\begin{array}{l}{[3,5]} \\
\text { Mbps }\end{array}$ & {$[2,4] \mathrm{Mbps}$} \\
\hline \multirow[t]{2}{*}{$\begin{array}{l}\text { Connectivity } \\
\text { (Edges) }\end{array}$} & Identifier & $\begin{array}{l}\text { Top } \\
\text { level } \\
(\mathrm{AS}) \\
\end{array}$ & $\begin{array}{c}\text { Bottom } \\
\text { level } \\
\text { (Nodes) }\end{array}$ \\
\hline & $\begin{array}{l}\text { MediumCo } \\
\mathrm{n}\end{array}$ & 5 & {$[20 / 2]=10$} \\
\hline $\begin{array}{l}\text { Cache } \\
\text { replacement } \\
\text { strategy }\end{array}$ & \multicolumn{3}{|c|}{$\begin{array}{l}\text { Least Recently Used (LRU) as most } \\
\text { commonly used because it replaces the } \\
\text { cache that has not been used for the longest } \\
\text { period of time [16]. }\end{array}$} \\
\hline Link failures & \multicolumn{3}{|c|}{$\begin{array}{l}50 \text { randomly link failures distributed } \\
\text { uniformly with interval [0, Simulation } \\
\text { time/10] }\end{array}$} \\
\hline $\begin{array}{l}\text { Content } \\
\text { distribution }\end{array}$ & \multicolumn{3}{|c|}{$\begin{array}{l}\text { Uniform content distribution is caches in } \\
\text { the core or centre of the network. }\end{array}$} \\
\hline $\begin{array}{l}\text { Simulation } \\
\text { time }\end{array}$ & \multicolumn{3}{|c|}{6 minutes (360 seconds) } \\
\hline
\end{tabular}

\subsection{Performance Metrics}

The studied performance metrics include Interest satisfaction ratio, cache hit ratio, hop count, delay and Interest retransmission. Interest satisfaction ratio is the ratio between 
received Data or known also as satisfied Interest packets and total generated Interests by all clients. Higher Interest satisfaction ratio means higher throughput. Some researchers call it as a Request success ratio [17]. Cache hit ratio is defined as the number of requests delivered by the nodes or cache hits, divided by the total number of requests, which include both cache hits and cache misses.

Hop count is defined as the number of network hops that the retrieved data packet travelled on the way back from producer application or cache [18]. Delay is defined as the average time taken starting from the time a consumer sends an Interest message until the time the consumer received the corresponding data [19]. Interest retransmission is defined as the number of unsatisfied Interests retransmitted by each consumer over total satisfied Interests [20]. We simulated few different scenarios to evaluate SAF performances to analyse the above-mentioned performance metrics.

\subsection{Simulation Scenarios}

The impact of having fixed as well as different number of servers to serve multiple clients was also studied in this experiment. In the $1^{\text {st }}$ scenario, we set 10 fixed servers to serve different number of clients, while we increased the number of servers accordingly in the $2^{\text {nd }}$ scenario. Table 2 depicts those scenarios.

Table 2: Scenario Design

\begin{tabular}{|l|l|l|l|l|l|}
\hline Scenario & \multicolumn{4}{|l|}{ Details } \\
\hline 1 & \multicolumn{4}{|l|}{$\begin{array}{l}\text { Different number of clients with fixed 10 } \\
\text { servers (in 6 minutes simulation time with } \\
50 \text { link failures) }\end{array}$} \\
\hline $\begin{array}{l}\text { Number of } \\
\text { clients }\end{array}$ & 20 & 40 & 60 & 80 & 100 \\
\hline & $\begin{array}{l}\text { Different number of clients and different } \\
\text { number of servers (in 6 minutes simulation } \\
\text { time with 50 link failures) }\end{array}$ \\
$\begin{array}{l}\text { Number of } \\
\text { clients }\end{array}$ & 20 & 40 & 60 & 80 & 100 \\
\hline $\begin{array}{l}\text { Number of } \\
\text { servers }\end{array}$ & 2 & 4 & 6 & 8 & 10 \\
\hline
\end{tabular}

\section{RESULTS AND DISCUSSION}

In brief, SAF and Best Route performance have been studied in two different scenarios. Figure 3 and Figure 4 show the results of Interest satisfaction ratio in which SAF outperforms Best Route in both scenarios. Both figures show similar trend in which the performance degrades as the number of clients with Interest increased. In average, the improvement made by SAF over Best Route was $11.7 \%$ for scenario 1 and $10.5 \%$ for scenario 2. However, the less number of servers have shown better results than having fixed number of servers, especially when there are only 2 or 4 servers available to serve the Interests (Figure 4). In order to fully understand the details of the Interest transmission in the NDN networks, we have run a simple experiment of small topology of 10 clients and 3 servers. We wish to investigate the flow of the Interests, either they reach the servers or cache nodes. From the observations, we can see that all of the available servers are trying to satisfy Interests from the same number the clients first, on one-to-one basis, and only then, the subsequent clients will get the respective contents from the nearest cache node(s). In this case, 3 servers will be responding to the Interests coming from the first 3 clients. Only then, the subsequent 7 clients will be getting the Data from the nearest cache node(s). It can be seen that having less number of servers results in higher Interest satisfaction rate. Nonetheless, further detailed experiments with different topologies are required to fully understand and verify such findings.

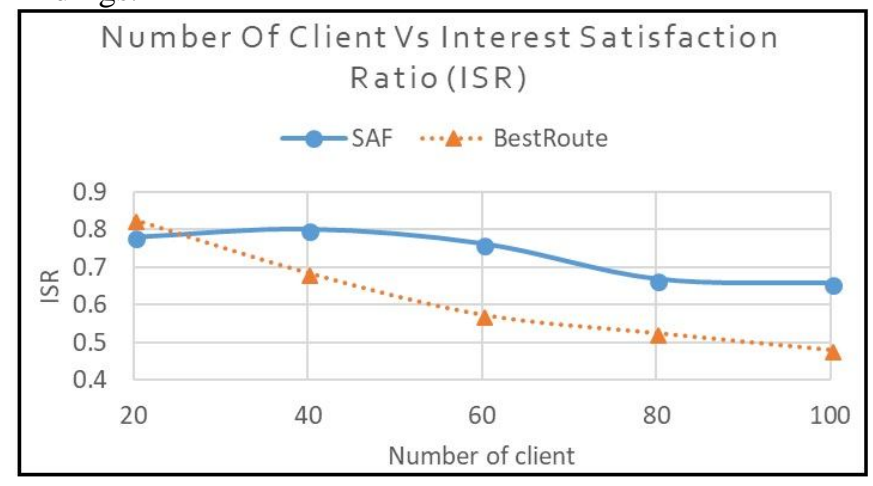

Figure 3: Average Interest Satisfaction Ratio with Fixed 10 Servers

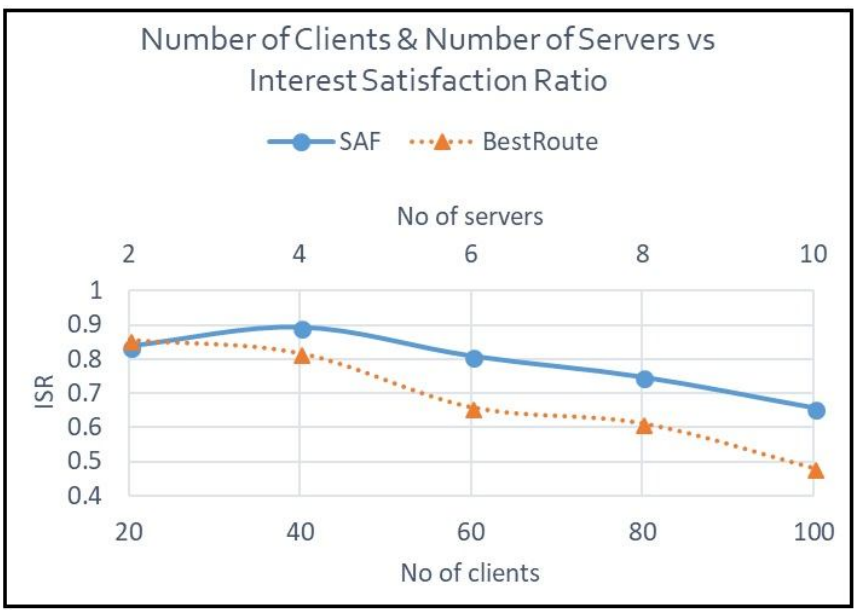

Figure 4: Average Interest Satisfaction Ratio with Different Number of Servers

Figure 5 and Figure 6 show the results of cache hit ratio in respective scenario. Total number of cache hits was recorded higher in scenario 2 although the graph shows its values decreases over time. The cache hit ratio value difference recorded between both scenarios was about 0.634. This situation occurs due to the reason explained in previous paragraph. In fixed number of servers' situation, as the number of clients increased, the more cache hits are recorded. 
For instance, for 40 clients, only 10 clients will be served by the servers and the rest of 30 clients will get their Interests replies from the cache nodes. Meanwhile, with 40 clients in scenario 2 , after 4 servers have replied to the Interests requests from 4 clients, the rest of 36 will be served by the cache node(s). Hence total cache hits is higher in scenario 2. More cache hits were recorded with SAF than Best Route in these experiments. The average cache hit recorded by SAF was about $29 \%$ higher for scenario 1 and about $27 \%$ higher for scenario 2.

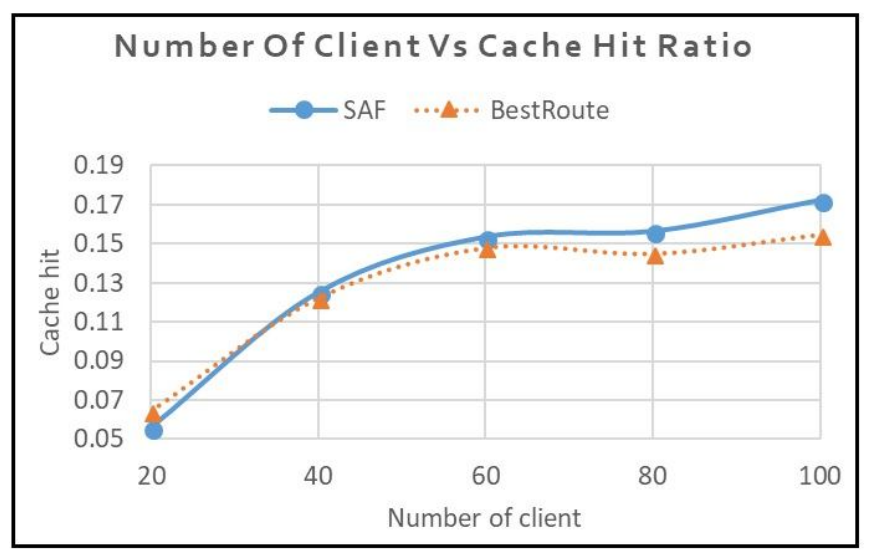

Figure 5: Average Cache Hit Ratio with Fixed 10 Servers

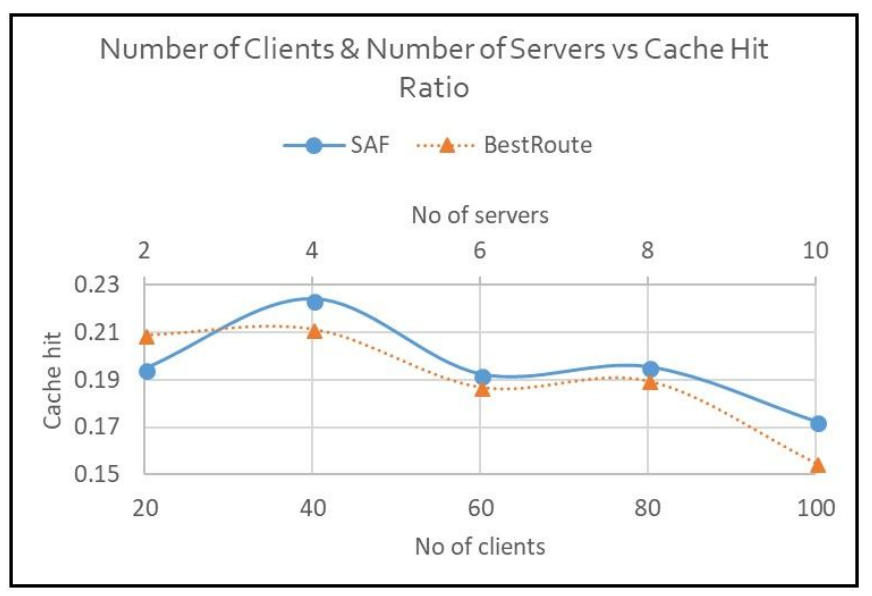

Figure 6: Average Cache Hit Ratio with Different

Number of Servers

Figure 7 and Figure 8 show that Best Route is having less average hop counts than that of SAF in both scenarios. This is the strength of Best Route algorithm, in which it always search for the paths with the least number of hops. Both scenarios have similar total average hop count values. The hop count values in scenario 1 is slightly higher than that in scenario at the beginning and consistently decreasing as the number of clients increased.

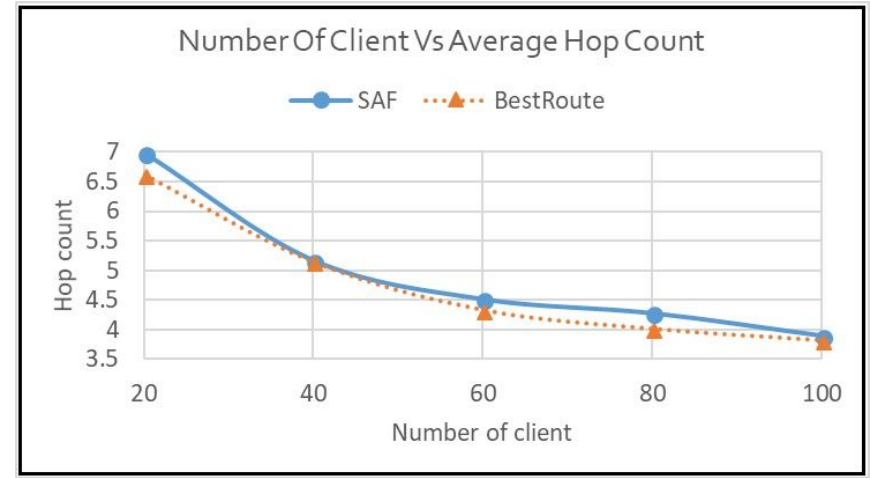

Figure 7: Average Hop Count with Fixed 10 Servers

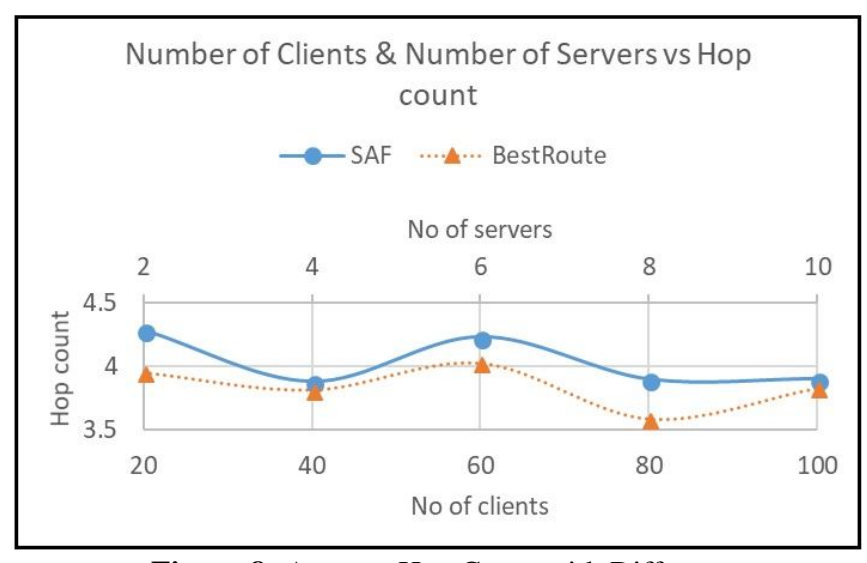

Figure 8: Average Hop Count with Different Number of Servers

The average delay trends for both scenarios are almost similar as shown in Figure 9 and Figure 10. SAF outperforms Best Route in both cases, that is by 37 seconds in scenario 1 and 28 seconds in scenario 2 . Less delay values were found when less number of allocated servers responded to the Interests from the clients. In other words, when more nearby cache nodes are available to respond to any Interests, the delay time can be reduced. For instance, if 40 clients are sending Interests requests, there are only 30 cache nodes are available to respond in scenario 1 , meanwhile there are 36 cache nodes are available in scenario 2 . This results in less delay time in the latter.

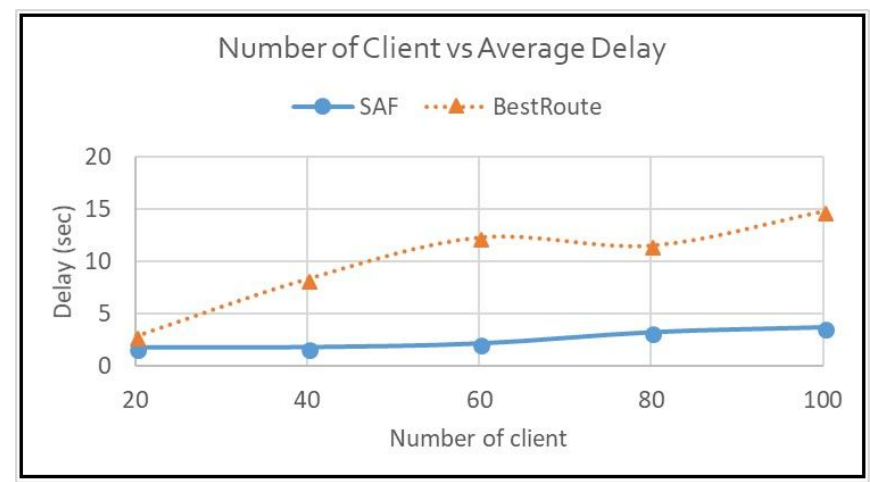

Figure 9: Average Delay with Fixed 10 Servers 


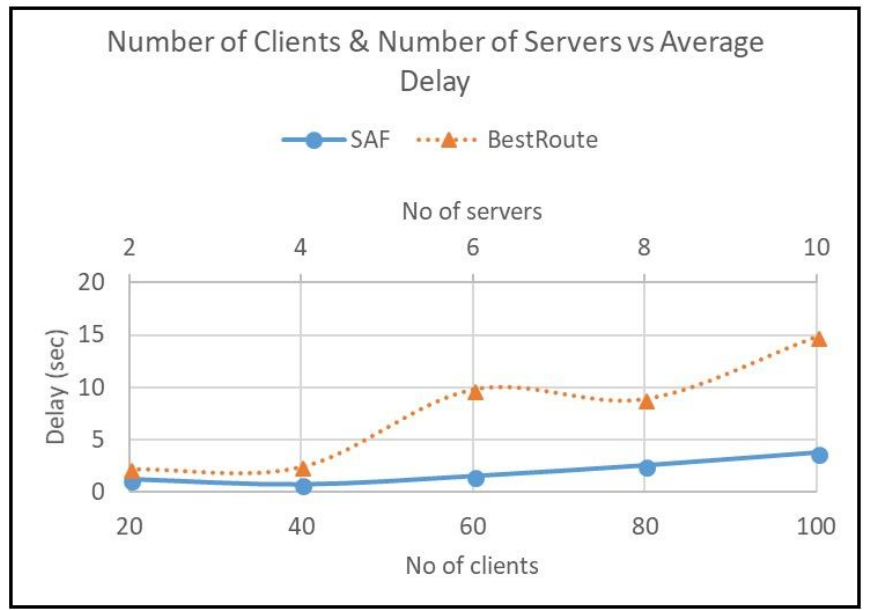

Figure 10: Average Delay with Different Number of Servers

The results on Interest retransmission is similar to those of average delays as shown in Figure 11 and Figure 12, with SAF performs better. SAF has around 0.63 less Interest retransmission value than Best Route in scenario 1, and 0.52 less value in scenario 2. The experiments show that less number of available dedicated servers to serve the requests results in better or smaller Interest retransmission value. The smaller Interest retransmission value shows that the Interests have been well responded to and hence the clients do not have to retransmit the same request.

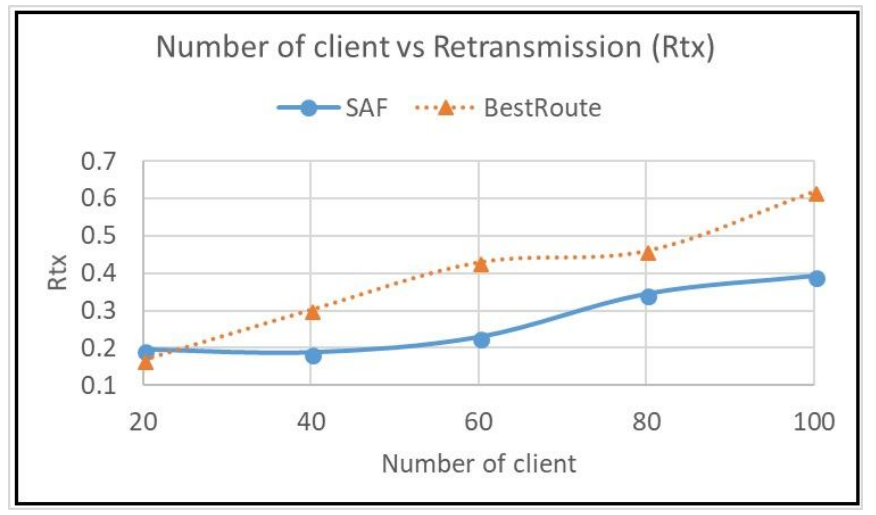

Figure 11: Average Retransmission with Fixed 10 Servers

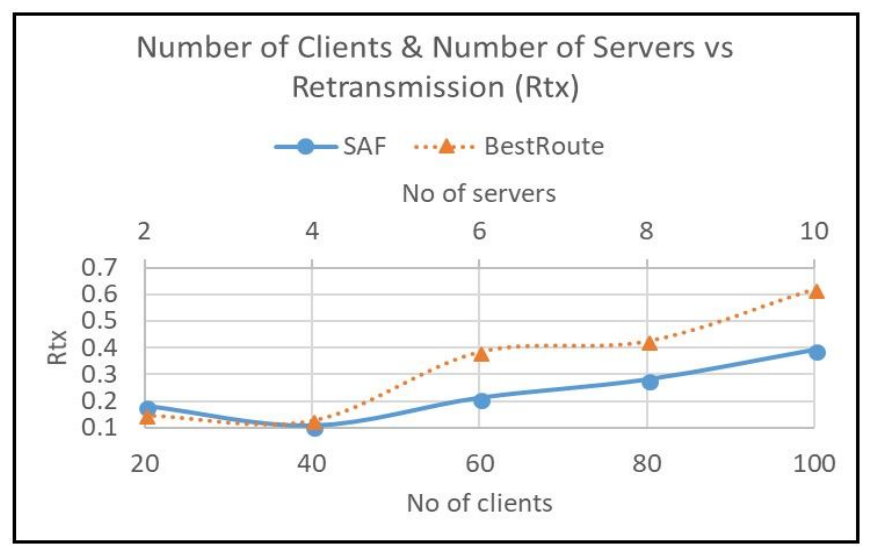

Figure 12: Average Retransmission with Different Number of Servers

\section{CONCLUSION AND FUTURE WORKS}

NDN closes the gap between application and network semantics by allowing the applications to name their data and have the network forward packets directly based on those names. In this paper, we have evaluated the performance of one of the adaptive strategies in NDN, namely Stochastic Adaptive Forwarding (SAF) against Best Route algorithm. The studied metrics include Interest satisfaction ratio, cache hit ratio, hop count, delay and Interest retransmission in two different environments. We also studied the impact of having fixed number of available servers (10 servers) versus networks with incrementing servers from 2 to 10 servers.

In general, SAF has outperformed Best Route except for the hop count metric as the latter has been designed to find the shortest route without evaluating the link performance or bandwidth. SAF, on the other hand evaluates multiple links performance and this has made SAF better in Interest satisfaction ratio, cache hit ratio, delay and Interest retransmission values. The trends for both average delay and average Interest retransmission are similar as they are related to each other. The high delay value can be caused by the timeout taken by the clients from sending the Interests when it reaches certain threshold, lower bandwidth link performance or repetitive Interest retransmission. The preliminary experiments have also shown that less number of servers would improve the Interest satisfaction ratio and average delay values due to many clients could retrieve the Data from the nearer cache nodes instead. Nonetheless, further detailed experiment is required to verify such result.

To conclude, SAF outperformed Best Route algorithm in many ways except for hop count. And it has been agreed though that hop count is not always the best performance indicator in forwarding strategy because it does not evaluate the link's performance such as bandwidth speed and others [2], [7]. For future work, the evaluation can be extended to a real life topology and to include other adaptive forwarding algorithms to further investigate SAF performance.

\section{REFERENCES}

1. L. Zhang, A. Afanasyev, J. Burke, V. Jacobson, KC Claffy, P. Crowley, C. Papadopoulos, L. Wang, and B. Zhang. Named data networking. SIGCOMM Comput. Commun. Rev. 44, 3, pp. 66-73, Jul. 2014. https://doi.org/10.1145/2656877.2656887

2. D. Posch, B. Rainer, and H. Hellwagner. SAF: Stochastic Adaptive Forwarding in Named Data Networking. IEEE/ACM Trans. Netw., vol. 25, no. 2, pp. 1089-1102, Apr. 2017.

https://doi.org/10.1109/TNET.2016.2614710

3. C. Yi, A. Afanasyev, I. Moiseenko, L. Wang, B. Zhang, and $\mathrm{L}$. Zhang. A case for stateful forwarding plane. Comput. Commun., vol. 36, no. 7, pp. 779-791, Apr. 2013.

https://doi.org/10.1016/j.comcom.2013.01.005 
4. C. Yi, A. Afanasyev, L. Wang, B. Zhang, and L. Zhang. Adaptive Forwarding in Named Data Networking. ACM SIGCOMM Comp. Communication Review, vol. 42, no. 3, pp. 62-67, Jul. 2012.

https://doi.org/10.1145/2317307.2317319

5. P. Moll, D. Posch, and H. Hellwagner. Investigation of push-based traffic for conversational services in Named Data Networking. IEEE International Conference on Multimedia \& Expo Workshops (ICMEW), pp. 315-320, 2017.

https://doi.org/10.1109/ICMEW.2017.8026212

6. Yuhang Ye, Brian Lee, R. Flynn, N. Murray, and Yuansong Qiao. HLAF: Heterogeneous-Latency Adaptive Forwarding strategy for Peer-Assisted Video Streaming in NDN. IEEE Symposium on Computers and Communications (ISCC), pp. 657-662, 2017.

https://doi.org/10.1109/ISCC.2017.8024603

7. A. Kerrouche, M. R. Senouci, and A. Mellouk. QoS-FS: A new forwarding strategy with QoS for routing in Named Data Networking. IEEE International Conference on Communications (ICC), pp. 1-7, 2016.

8. J. Cao, D. Pei, Z. Wu, X. Zhang, B. Zhang, L. Wang, and $\mathrm{Y}$. Zhao. Improving the freshness of NDN forwarding states. IFIP Networking Conference (IFIP Networking) and Workshops, pp. 189-197, 2016. https://doi.org/10.1109/IFIPNetworking.2016.7497213

9. H. Qian, R. Ravindran, G. Q. Wang, and D. Medhi. Probability-based adaptive forwarding strategy in named data networking. IFIP/IEEE International Symposium on Integrated Network Management (IM 2013), pp. 1094-1101, 2013.

10. A. Udugama, X. Zhang, K. Kuladinithi, and C. Goerg. An On-demand Multi-Path Interest Forwarding strategy for content retrievals in CCN. 2014 IEEE Network Operations and Management Symposium (NOMS), pp. 1-6, 2014.

11. D. Nguyen, M. Fukushima, K. Sugiyama, and A. Tagami. Efficient multipath forwarding and congestion control without route-labeling in $\mathrm{CCN}$. 2015 IEEE International Conference on Communication Workshop (ICCW), pp. 1533-1538, 2015.

12. J. Yao, B. Yin, X. Tan, and X. Jiang. A POMDP framework for forwarding mechanism in named data networking. Comput. Networks, vol. 112, pp. 167-175, Jan. 2017. https://doi.org/10.1016/j.comnet.2016.11.005

13. K. Lei, J. Yuan, and J. Wang. MDPF: An NDN Probabilistic Forwarding Strategy Based on Maximizing Deviation Method. 2015 IEEE Global Communications Conference (GLOBECOM), pp. 1-7, 2015.

14. A. Afanasyev, J. Shi, B. Zhang, L. Zhang, I. Moiseenko, Y. Yu, W. Shang, Y. Huang, J. P. Abraham, S. DiBenedetto, C. Fan, C. Papadopoulos, D. Pesavento, G. Grassi, G. Pau, H. Zhang, T. Song, H. Yuan, H. B.
Abraham, P. Crowley, S. O. Amin, V. Lehman, and L. Wang. NFD developers guide. NDN Project, Tech. Rep. NDN-0021, Jul. 2014.

15. ndnSIM Documentation. http://ndnsim.net/2.5/. Accessed July 2018.

16. Y. Thomas, G. Xylomenos, C. Tsilopoulos, and G. C. Polyzos. Object-oriented Packet Caching for ICN. $2^{\text {nd }}$ ACM Conference on Information-Centric Networking, pp. 89-98, Oct. 2015. https://doi.org/10.1145/2810156.2810172

17. C. Bian, T. Zhao, X. Li, and W. Yan. Boosting named data networking for data dissemination in urban VANET scenarios. Veh. Commun., vol. 2, no. 4, pp. 195-207, Oct. 2015.

18. Obtaining metrics - ndnSIM documentation. http://ndnsim.net/current/metric.html. Accessed May 2018.

19. E. Kalogeiton, T. Kolonko, and T. Braun. A topology-oblivious routing protocol for NDN-VANETs. Annals of Telecommunications, vol 73, no 9-10, pp 577-587, Oct 2018. https://doi.org/10.1007/s12243-018-0661-4

20. M. Amadeo, A. Molinaro, C. Campolo, M. Sifalakis, and C. Tschudin. Transport layer design for named data wireless networking. IEEE Conference on Computer Communications Workshops (INFOCOM WKSHPS), pp. 464-469, 2014. https://doi.org/10.1109/INFCOMW.2014.6849276 